\title{
Natural history of ovarian endometrioma in pregnancy
}

\author{
Katie Pateman, Francesca Moro, Dimitrios Mavrelos, Xulin Foo, Wee-Liak Hoo and Davor Jurkovic ${ }^{*}$
}

\begin{abstract}
Background: Ovarian endometriomas are classified as benign ovarian lesions. During pregnancy endometriomas may undergo major morphological changes which are referred to as 'decidualisation'. Decidualised ovarian endometrioma may resemble malignant ovarian tumours on ultrasound examination. The aim was to study variations in the morphology and size of ovarian endometriomas diagnosed on ultrasound during pregnancy.
\end{abstract}

Methods: We searched our database to identify pregnant women who were diagnosed with ovarian endometriomas on ultrasound in order to study the effect of pregnancy on their morphological characteristics. In women who underwent serial scans during pregnancy we examined the changes in the size of endometriomas with advancing gestation.

Results: Twenty four patients with a total of 34 endometriomas were included in the analysis. All women were managed expectantly during pregnancy. On the first ultrasound scan $29 / 34$ (85.3\%, 95\% Cl 73.4 - 97.2) endometriomas appeared unilocular with fine internal echoes ('ground glass' contents) and they were poorly vascularised on Doppler examination. 1/34 (2.9\% 95\% Cl 0.0 - 8.5) endometrioma was multilocular, with regular margins, 'ground glass' contents and it was also poorly vascularised. 4/34 (11.8\%, 95\% Cl 1.0 - 22.6) had sonographic features suggestive of decidualisation such as thick and irregular inner wall, papillary projections and highly vascular on Doppler examination. The endometriomas showed a tendency to decrease in size during pregnancy.

Conclusions: Pregnancy has a major effect on the size and morphological appearances of ovarian endometriomas. Rapid regression of decidualised endometriomas is a helpful feature which could be used to confirm their benign nature.

Keywords: Endometrioma, Decidualisation, Ovarian cyst, Pregnancy

\section{Background}

The number of women diagnosed with ovarian cysts during pregnancy has increased in recent years mainly due to the widespread use of ultrasound in the first trimester. It has been reported that adnexal cysts can be seen on ultrasound scan in $4.1 \%$ to $24.9 \%$ of pregnant women [1,2]. Ovarian endometriomas account for $4-5 \%$ of ovarian cysts diagnosed in early pregnancy [3]. The appearances of endometriomas on ultrasound are variable, ranging from unilocular cysts with low level echoes ('ground glass' echogenicity) $[4,5]$ to solid looking tumours [4-6]. Despite the variability in their appearances endometriomas are relatively easy to classify correctly on ultrasound examination with the reported sensitivity and specificity being as high as $92 \%$ and $97 \%$ respectively $[4,5,7-9]$. Hormonal changes

\footnotetext{
* Correspondence: davor.jurkovic@uclh.nhs.uk

Department of Obstetrics and Gynaecology, University College London Hospital, 235 Euston Road, London NW1 2BU, UK
}

associated with the pregnancy may cause alterations in the sonographic appearances of endometriomas which are referred to as decidualisation. Decidualisation is the process of endometrial change caused by high progesterone levels which increases glandular epithelial secretion, accumulation of glycogen and stromal vascularity. These changes create conditions which facilitate implantation and development of early gestation. Formation of ectopic decidua (deciduosis) during pregnancy is a well-documented phenomenon that is caused by the effect of progesterone on ectopic endometrium, such as in foci of endometriosis [10]. Decidualised endometriomas may develop extensive intraluminal papillary projections with increased blood flow which are similar to malignant ovarian tumours [11-13].

The process of decidualisation of ovarian endometrioma during pregnancy is well documented. Although the ultrasound features of decidualisation can mimic ovarian cancer 
this appears to be a transient phenomenon. Decidualised endometrioma are usually surgically removed. We found only ten women with decidualised endometriomas who were managed expectantly during pregnancy, one had a first trimester miscarriage and so was not included in these results. Eight of them underwent ovarian cystectomy or oophorectomy at the time of Caesarean section. In all eight cases the diagnosis of benign decidualised ovarian endometrioma was confirmed on histopathology [14-19].

Decidualised ovarian endometrioma may resemble malignant ovarian tumours on ultrasound examination which can cause anxiety to women and lead to unnecessary and potentially harmful surgical interventions. There are several reports in the literature describing appearances of decidualised ovarian endometrioma prior to surgery [6,10,14,15,19-25]. Little is known, however, about the proportion of endometriomas that undergo decidualisation and their other changes during pregnancy. The aim of this article is to present an overview of the sonographic characteristics of ovarian endometriomas in pregnancy and provide more information about their natural history.

\section{Methods}

We searched the database at the Early Pregnancy and Gynaecological Diagnostic and Outpatient Treatment Unit at University College London Hospital, London, UK to identify all pregnant women with a conclusive diagnosis of ovarian endometrioma. In all cases the diagnosis was confirmed by expert operators who had more than 10 years of experience in gynaecological ultrasound. The diagnosis was made using 'pattern recognition technique' which defined an endometrioma as a cyst typically located in the centre of the ovary with 'ground glass' echogenicity and surrounded by healthy ovarian tissue. On palpation the endometriomas were often adhered to the surrounding pelvic structures and on Doppler examination they were poorly vascularised [5] (Figure 1).

Decidualised endometrioma were diagnosed in women who presented with unilocular cysts containing hyperechoic fluid and irregular internal wall with prominent echogenic papillary projections [26]. The papillary projections were typically highly vascular on Doppler examination (Figure 2).
Maternal demographic data, indications for examinations, clinical symptoms and ultrasound findings were all recorded.

The site and morphology of ovarian endometriomas were recorded and the cysts were measured in three orthogonal planes. The surrounding ovarian tissue and ovarian capsule were not included in the measurements of the ovarian cyst. The volume of the cyst was calculated using the formula for an ellipsoid (4/3 $\Pi$ abc, where $a, b$, and $c$ are the semi-axes of the cyst). The side and morphology of the corpus luteum was also recorded.

The number of follow-up scans and the time intervals between scans were also recorded.

Ethical approval was sought from, and approved by, the Joint Research Office at University College London Hospital who deemed that full ethical approval was not required.

\section{Statistical analysis}

The Kolmogorov-Smirnov test was used to test for normal distribution. Age, the time interval between ultrasound scans, ovarian cyst dimension and volume were not normally distributed and was expressed as median (interquartile range (IQR)). We used the Mann-Whitney $U$-test to compare the medians of non-normally distributed variables. Proportions were expressed as percentage (95\% CI). We used the chi-square test and Fisher's exact test to compare proportions.

\section{Results}

We identified 24 pregnant women who were diagnosed with ovarian endometriomas in pregnancies between January 2009 and May 2013. The median maternal age was 35 years (IQR 32 - 37 years). 22/24 (92.0\%, 95\% CI 81.2 $100)$ women were nulliparous and of these $11 / 22(50.0 \%$, 95\% CI 29.1 - 70.9) were primigravid. Two remaining parous women were a primipara and a multipara.

20/24 (83.3\%, 95\% CI 68.4 - 98.2) women attended for their initial ultrasound scan in the first trimester of pregnancy, whilst the remaining 4/24 (16.7\%, 95\% CI 1.8 31.6) women had their first scans between 13 - 19 weeks' gestation. The median gestational age at presentation
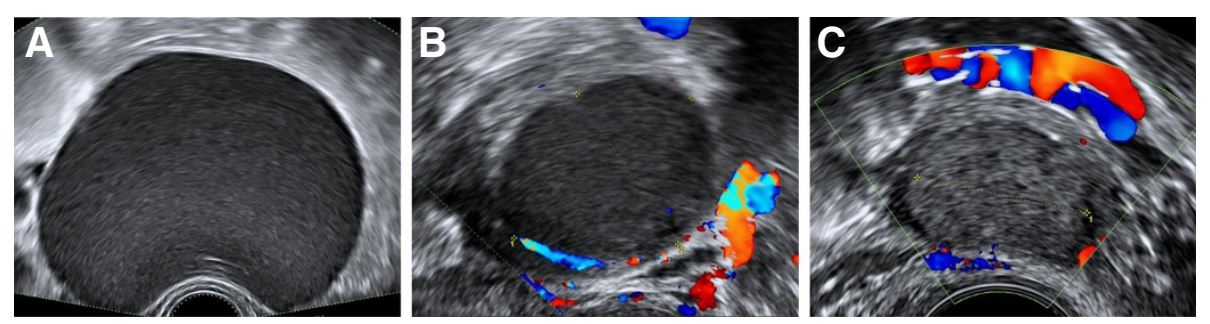

Figure 1 The sonographic appearances of typical endometrioma. Unilocular cysts with "ground glass" contents [A] and are poorly vascular or avascular on colour Doppler examination [B and C]. 

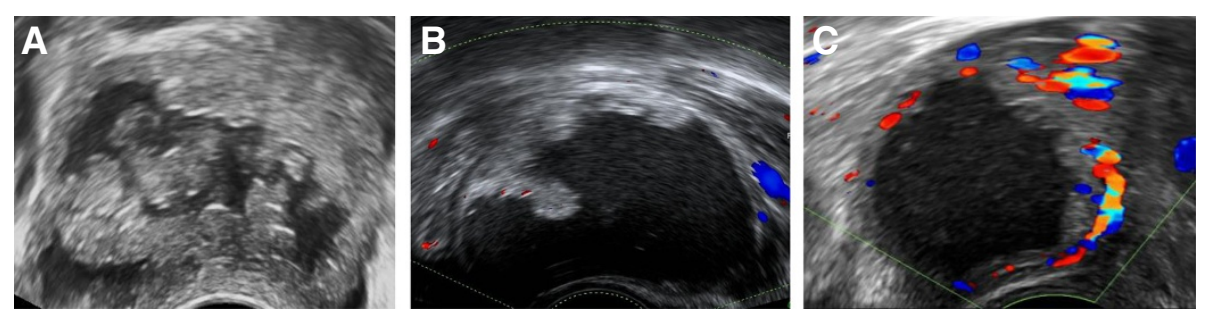

Figure 2 Sonographic appearances of decidualised endometrioma. Unilocular cysts containing hyperechoic fluid and irregular internal wall with prominent echogenic rounded papillary projections [A]. The papillary projections were typically highly vascular on Doppler examination [B and C].

was $9^{+3}$ weeks (IQR $6-12^{+3}$ ). Indications for ultrasound scans at presentation were: lower abdominal pain in $8 / 24$ (33.3\%, 95\% CI 14.4 - 52.2), vaginal bleeding in $6 / 24$ (25.0\%, 95\% CI 7.7 - 42.3), previous history of miscarriage in 5/24 (20.8\%, 95\% CI 4.6 - 37.0), and incidental finding of ovarian cyst on the routine 12 weeks' scan in $5 / 24$ (20.8\%, 95\% CI 4.6 - 37.0) women.

There were 34 cysts in total: $15 / 24(62.5 \%, 95 \%$ CI 43.1 - 81.9) women had single unilateral endometrioma, 5/24 (20.8\%, 95\% CI 4.6 - 37.0) had multiple unilateral cysts and 4/24 (16.7\%, 95\% CI 1.8 - 31.6) had bilateral endometriomas. 9/24 (37.5\%, 95\% CI 18.1 - 56.9) women were surgically diagnosed with endometriosis prior to their pregnancies.

On the initial scan 29/34 (85.3\%, 95\% CI 73.4-97.2) cysts appeared unilocular with fine internal echoes ("ground glass" contents) and they were either avascular or poorly vascular on Doppler examination. 1/34 (2.9\% $95 \%$ CI 0.0 - 8.5) cysts were multilocular and $4 / 34$
(11.8\%, 95\% CI 1.0 - 22.6) cysts had sonographic features suggestive of decidualisation.

In 15 women with unilateral cysts, the corpus luteum was present in the contralateral ovary in $11 / 15$ (73.3\%, 95\% CI 50.9 - 95.7) cases and in the ipsilateral ovary in 4/15 (26.7\%, 95\% CI 4.3 - 49.1) cases.

22/34 (64.7\%, 95\% CI 48.6 - 80.8) cysts decreased in size during pregnancy, 9/34 (26.5\%, 95\% CI 11.7 - 41.3) were unchanged during follow up and 3/34 (8.8\%, 95 CI 0.0 - 18.3) cysts increased in size (Figure 3 ).

The median volume of the cysts at initial examination was $14.0 \mathrm{ml}$ (IQR 3.0 - 30.0). The median rate of volume change of all endometriomas during the study period was $-0.5 \mathrm{ml} /$ week (IQR -2.8 to 0 ) or $-5.2 \%$ per week (IQR -10.9 to 0$)$.

There was no significant difference between decidualised and non-decidualised cysts in median volume at presentation $(54.0 \mathrm{ml}$ [IQR 23.5 to 77.0$]$ vs. $12.5 \mathrm{ml}$ [IQR 3.0 to 26.0], p=0.105), absolute percent change

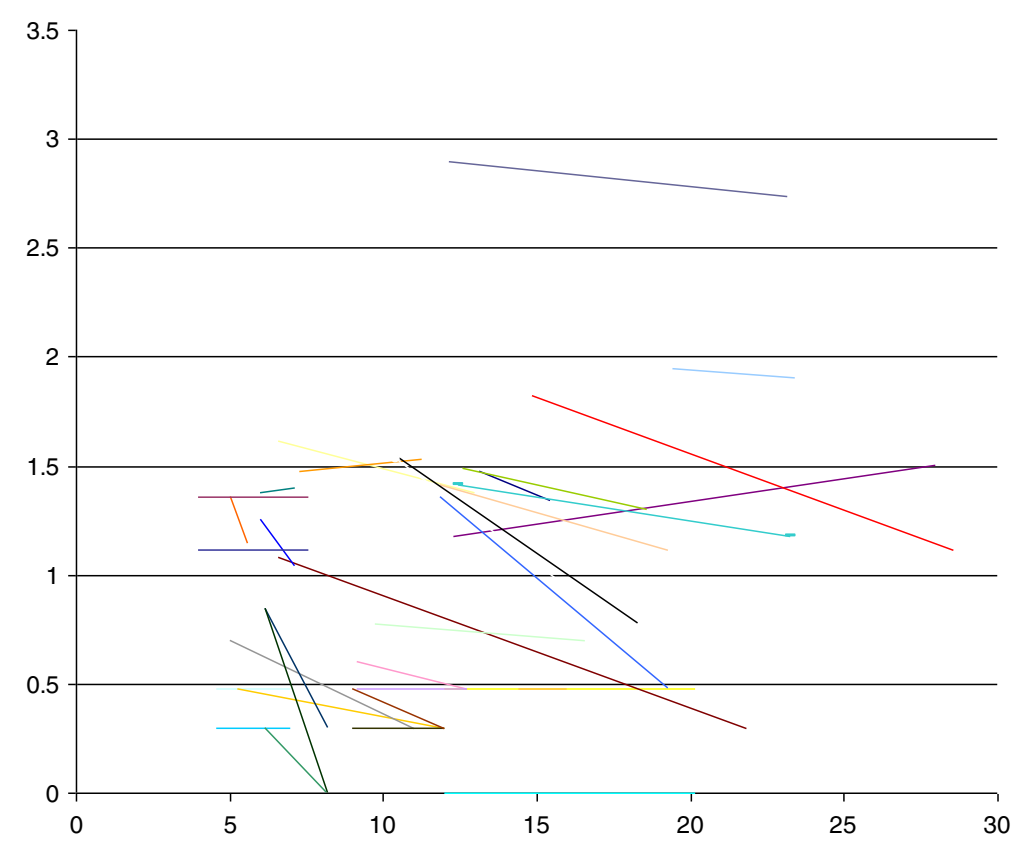

Figure 3 Change in ovarian endometrioma volume over advancing gestation. Y axis: Log of cyst volume (mls). X axis: Gestation (weeks). 
$(-29.1 \%$ [IQR -70.8 to -11.0 ] vs. $-32.1 \%$ [ -52.5 to 0.0 ], $\mathrm{p}=0.661)$ rate of change $(-4.2 \%$ per week $[-6.3$ to -2.3$]$ vs. $-5.2 \%$ per week [IQR -11.25 to 0.0$], \mathrm{p}=0.897$ ).

All women were managed expectantly during the pregnancy. One woman diagnosed with a decidualised endometrioma had ovarian cystectomy at the time of her Caesarean section and the histology report confirmed the pre-operative ultrasound diagnosis. 4/24 women who attended for postnatal scan had a reduction in the cysts volume compared to the initial measurements between $45.9 \%$ and $88.5 \%$.

\section{Discussion}

Our study has shown that pregnancy has a major effect on the biological behaviour of ovarian endometriomas. The majority of cysts decrease in size during pregnancy with the rate of regression being particularly high in decidualised endometriomas. This is in agreement with a widely held view that pregnancy has beneficial effect on endometriosis. High progesterone levels produced during pregnancy and temporary cessation of menstrual cycles and induce apoptosis are probably the main factors causing regression of endometriosis $[27,28]$.

We also found that $12 \%$ of endometrioma undergo decidualisation. Our findings are in agreement with a retrospective analysis reported by Ueda et al. [18] who also found signs of decidualisation in $12 \%$ of ovarian endometrioma during pregnancy. There are no published reports of decidualised endometriomas occurring outside of pregnancy for instance occurring in women receiving exogenous progesterones in form of pill, hormone implants or levonorgestrel-releasing intrauterine system. This could be explained by the high placental progesterone production which exceeds the amounts given during exogenous therapy. In addition, increased tissue perfusion and oedema caused by pregnancy could contribute to the formation of intraluminal mucosal folds and vascular papillary projections [29].

The reported prevalence of endometriomas in pregnancy is between $0.2 \%$ and $0.3 \%$ [2,3]. Despite their relatively rarity they account for approximately a quarter of all surgical interventions for ovarian cysts detected during pregnancy [3].

A review of the literature showed that there is limited information regarding behaviour of endometriomas during pregnancy (Table 1). The first report of decidualised endometrioma in pregnancy was published by Miyakoshi in 1998 [10]. Since then, a number of further cases have been reported [6,14-26,30]. These reports provided descriptions of ultrasound features of decidualised endometriomas during pregnancy. In many cases decidualised endometriomas resembled ovarian malignancy. 28/31 (90\%) women were offered surgical intervention, but two women opted for expectant management instead. In those who went ahead with the operation there were 9/26 (35\%) laparotomies, 2/26 (8\%) laparoscopies and the surgical approach was not described in the remaining fifteen cases. In 9/14 women with reported pregnancy outcomes following surgery there were $8 / 9$ (89\%) live births at term and one woman suffered a second trimester miscarriage. Although these findings were reassuring other studies have shown that surgical intervention in pregnancy may be associated with increased risks to maternal and fetal health [31]. A recent meta-analysis showed that risk of miscarriage is significantly higher in women undergoing laparoscopic compared to open appendicectomy in pregnancy [32]. In recent years laparoscopy has become the method of choice for excision of ovarian cysts in both nonpregnant and pregnant women. Although there is no specific data regarding the risks of laparoscopic cystectomy/ oophorectomy in pregnancy, it is possible that they could be similar to laparoscopic appendicectomy. However, the risk of laparoscopic appendicectomy may be higher due to concomitant infection. In addition women with suspected ovarian cancer undergoing surgery are also likely to suffer psychological harm due to anxiety and stress [33].

Our data show that expectant management of decidualised endometrioma is likely to be safe with a fast regression rate. We have also found that decidualised endometrioma tend to regress particularly fast. This is a reassuring feature which may be used to help to avoid unnecessary interventions due to difficulties in differential diagnosis between decidualised ovarian endometrioma and ovarian cancer on the initial scan. Our findings are also in agreement with a recent published study by Mascilini et al. [19], who also noted that the highly vascularised papillary projections are more rounded with a smooth contour.

Decidualised endometriomas tend to resemble borderline ovarian tumours which also contain multiple papillary projections and tend to be surrounded by normal ovarian parenchyma. Borderline tumours typically increase in size during follow up, whilst decidualised endometrioma show the opposite trend. Other ultrasonographic features of endometriosis, such as obliteration of the pouch of Douglas and deep infiltrating endometriotic nodules [34] may also help facilitate the difference between borderline ovarian tumours and decidualised endometriomas. Additional tests which are used to differentiate between benign and malignant cysts such as serum CA125 are not helpful in pregnancy. Serum CA125 levels in particular are significantly higher in pregnant women which may lead to a false positive diagnosis of ovarian cancer during pregnancy [35]. Other complications such as ovarian torsion are unlikely to occur in ovarian endometriomas which tend to be firmly adhered to the surrounding pelvic structures. 
Table 1 The management of decidualised endometriomas in the literature

\begin{tabular}{|c|c|c|c|c|c|c|}
\hline Author & $\begin{array}{l}\text { No of } \\
\text { cases }\end{array}$ & Management & Surgical approach & Intervention & $\begin{array}{l}\text { Gestation at } \\
\text { intervention }\end{array}$ & Pregnancy outcome \\
\hline \multirow[t]{2}{*}{ Sammour } & 2 & Surgical & $\begin{array}{l}\text { Laparoscopy converted } \\
\text { to laparotomy }\end{array}$ & Oophorectomy & $16 / 40$ & NVD at term \\
\hline & & Surgical & Laparoscopy & Salpingo-oophorectomy & NS & NVD at term \\
\hline Tazegul & 1 & Surgical & NS & Cystectomy & $12 / 40$ & NS \\
\hline Poder & 1 & Expectant & & & $38 / 40$ & $\begin{array}{l}\text { Caesarean section and } \\
\text { salpingo-oophorectomy }\end{array}$ \\
\hline Guerriero & 1 & Expectant & & & $37 / 40$ & $\begin{array}{l}\text { Caesarean section, frozen } \\
\text { section, cystectomy }\end{array}$ \\
\hline Fruscella & 1 & Surgical & Laparotomy & Cystectomy & $18 / 40$ & Caesarean section at term \\
\hline Miyakoshi & 1 & Surgical & Laparotomy & Salpingo-oophorectomy & $22 / 40$ & NS \\
\hline Tanaka & 1 & Surgical & NS & Cystectomy & $12 / 40$ & NS \\
\hline \multirow[t]{3}{*}{ Barbieri } & 3 & Surgical & Laparoscopy & Frozen section, cystectomy & $14 / 40$ & NVD at term \\
\hline & & $\begin{array}{l}\text { Expectant (declined } \\
\text { surgery 24/40) }\end{array}$ & & & $36 / 40$ & $\begin{array}{l}\text { Concomitant Caesarean section } \\
\text { and frozen section, cystectomy }\end{array}$ \\
\hline & & $\begin{array}{l}\text { Expectant (declined } \\
\text { surgery at 10/40) }\end{array}$ & & Expectant & $10 / 40$ & Miscarriage \\
\hline Iwamoto & 1 & Surgical & Laparotomy & Salpingo-oophorectomy & $22 / 40$ & NS delivery at term \\
\hline \multirow[t]{2}{*}{ Yoshida } & 2 & Surgical & Laparotomy & Oophorectomy & $28 / 40$ & NS delivery at term \\
\hline & & Surgical & Laparotomy & Cystectomy & $16 / 40$ & NS \\
\hline Sayasneh & 1 & Expectant & & Expectant & & NVD at term \\
\hline \multirow[t]{3}{*}{ Machida } & 3 & Surgical & Laparotomy & Salpingo-oophorectomy & $19 / 40$ & PROM - TOP 21/40 \\
\hline & & Surgical & Laparotomy & Salpingo-oophorectomy & $14 / 40$ & NVD at term \\
\hline & & Surgical & Laparotomy & Frozen section, cystectomy & $14 / 40$ & NVD at term \\
\hline Asch & 1 & Surgical & NS & NS & NS & NS \\
\hline \multirow[t]{3}{*}{ Ueda } & 3 & Surgical & NS & Salpingo-oophorectomy & NS & NS \\
\hline & & Surgical & NS & Salpingo-oophorectomy & NS & NS \\
\hline & & Expectant & & & & NS \\
\hline \multirow[t]{2}{*}{ Mascilini } & $13^{\S}$ & Surgical & NS & NS & NS & NS \\
\hline & 5 & Expectant & & & & $\begin{array}{l}\text { Concomitant Caesarean } \\
\text { section and NS cystectomy } \\
\text { or oophorectomy }\end{array}$ \\
\hline
\end{tabular}

NS not specified, NVD normal vaginal delivery, PPROM premature rupture of membranes, TOP termination of pregnancy.

${ }^{\S}$ The case by Fruscella et al. is also included in this total.

The limitation of our study was its retrospective nature with no structured follow-up. The majority of patients did not attend for a post-natal scan and therefore the changes following delivery could not be assessed. In addition, the proportion of decidualised cysts may have been overestimated as women with small non-decidualised endometriomas are less likely to be referred for specialised gynaecology examination. The majority of women were managed conservatively and histological confirmation of ultrasound diagnosis is lacking in most cases.

Future prospective studies should provide more information about the natural history of endometriomas in pregnancy and pregnancy outcomes following surgical interventions. More information is also needed regarding recurrence rates following uncomplicated pregnancy and delivery.

\section{Conclusions}

Pregnancy has a major effect on the size and morphological appearances of ovarian endometriomas. The decidualisation rate of ovarian endometriomas diagnosed in pregnancy in our cohort was $12 \%$. Rapid regression of decidualised endometriomas is a helpful feature which could be used to confirm their benign nature.

\section{Abbreviations}

CA: 125 Cancer antigen 125; Cl: Confidence intervals; IQR: Interquartile range; ML: Millilitres; NS: Not specified; NVD: Normal vaginal delivery; PROM: Premature rupture of membranes; TOP: Termination of pregnancy. 


\section{Competing interests}

The authors declare that they have no competing interests.

\section{Authors' contributions}

KP designed the study protocol and the data collection proforma, collected the data, performed statistical analysis and drafted the manuscript. FM aided data collection and was involved with drafting the manuscript. DM made substantial contributions to statistical analysis and interpretation of data. XF and WLH were involved with data collection. DJ conceived the study concept participated in its design and co-ordination, helped with data analysis and finalising the manuscript. All authors approved the final manuscript.

\section{Authors' information}

Gynaecology Diagnostic and Outpatient Treatment Unit Lower ground floor, EGA Wing, University College London Hospital 235 Euston Road, London, NW1 2BU, UK.

\section{Acknowledgements}

The authors would like to thank all our receptionists, nurses and clinic staff who help provide support for all our patients coming to our service.

Received: 27 March 2014 Accepted: 6 October 2014

Published: 15 October 2014

\section{References}

1. Hill LM, Connors-Beatty DJ, Nowak A, Tush B: The role of ultrasonography in the detection and management of adnexal masses during the second and third trimesters of pregnancy. Am J Obstet Gynecol 1998, 179:703.

2. Yazbek J, Salim R, Woelfer B, Aslam N, Lee CT, Jurkovic D: The value of ultrasound visualization of the ovaries during the routine 11-14 weeks nuchal translucency scan. Eur J Obstet Gynecol Reprod Biol 2007, 132:154-158.

3. Condous G, Khalid A, Okaro E, Bourne T: Should we be examining the ovaries in pregnancy? Prevalence and natural history of adnexal pathology detected at first-trimester sonography. Ultrasound Obstet Gynecol 2004, 24:62-66.

4. Timmerman D, Schwarzler P, Collins WP, Claerhout F, Coenen M, Amant F, Vergote I, Bourne TH: Subjective assessment of adnexal masses with the use of ultrasonography: an analysis of interobserver variability and experience. Ultrasound Ostet Gynecol 1999, 13:11-16.

5. Van Holsbeke C, Van Calster B, Guerriero S, Savelli L, Paladini D, Lissoni AA, Czekierdowski A, Fischerova D, Zhang J, Mestdagh G, Testa AC, Bourne T, Valentin L, Timmerman D: Endometriomas: their ultrasound characteristics. Ultrasound Obstet Gynecol 2010, 35:730-740.

6. Asch E, Levine D: Variations in appearance of endometriomas. J Ultrasound Med 2007, 26:993-1002.

7. Valentin L: Pattern recognition of pelvic masses by gray-scale ultrasound imaging: the contribution of Doppler ultrasound. Ultrasound Obstet Gynecol 1999, 14:338-47.

8. Jermy K, Luise C, Bourne T: The characterization of common ovarian cysts in premenopausal women. Ultrasound Obstet Gynecol 2001, 17:140-4.

9. Milad MP: Cohen L Preoperative ultrasound assessment of adnexal masses in premenopausal women. Int J Gynaecol Obstet 1999, 66(2):137-41.

10. Miyakoshi K, Tanaka M, Gabionza D, Takamatsu K, Miyazaki T, Yuasa Y, Mukai $M$, Yoshimura $Y$ : Decidualized ovarian endometriosis mimicking malignancy. Am J Roentgenol 1998, 171:1625-1626.

11. Alcázar JL, Mercé LT, Laparte C, Jurado M, López-García G: A new scoring system to differentiate benign from malignant adnexal masses. Am J Obstet Gynecol 2003, 188:685-692

12. Eskenazi $B$, Warner $M$, Bonsignore $L$, Olive $D$, Samuels $S$, Vercellini $P$ : Validation study of nonsurgical diagnosis of endometriosis. Fertil Steril 2001, 76:929-935.

13. Patel MD, Feldstein VA, Chen DC, Lipson SD, Filly RA: Endometriomas: diagnostic performance of US. Radiology 1999, 210:739-745.

14. Poder L, Coakley FV, Rabban JT, Goldstein RB, Aziz S, Chen LM: Decidualized endometrioma during pregnancy: recognizing an imaging mimic of ovarian malignancy. J Comput Assist Tomogr 2008, 32:555-558.

15. Barbieri M, Somigliana E, Oneda S, Ossola MW, Acaia B, Fedele L: Decidualized ovarian endometriosis in pregnancy: a challenging diagnostic entity. Hum Reprod 2009, 24:1818-1824.
16. Sayasneh A, Naji O, Abdallah Y, Stalder C, Bourne T: Changes seen in the ultrasound features of a presumed decidualised ovarian endometrioma mimicking malignancy. J Obstet Gynaecol 2012, 32:807-11.

17. Guerriero S, Ajossa S, Piras S, Parodo G, Melis GB: Serial ultrasonographic evaluation of a decidualized endometrioma in pregnancy. Ultrasound Obstet Gynecol 2005, 26:304-6.

18. Ueda Y, Enomoto T, Miyatake T, Fujita M, Yamamoto R, Kanagawa T, Shimizu H, Kimura T: A retrospective analysis of ovarian endometriosis during pregnancy. Fertil Steril 2010, 94:78-84.

19. Mascilini F, Moruzzi C, Giansiracusa C, Guastafierro F, Savelli L, De Meis L, Epstein E, Timor-Tritsch IE, Mailath-Pokorny M, Ercoli A, Exacoustos C, Benacerraf BR, Valentin L, Testa AC: Imaging of gynecological disease: clinical and ultrasound characteristics of decidualized endometriomas surgically removed during pregnancy. Ultrasound Obstet Gynecol 2014, 4. doi:10.1002/uog.13323.

20. Tanaka YO, Shigemitsu S, Nagata M, Shindo M, Okamoto Y, Yoshikawa H, Itai Y: A decidualized endometrial cyst in a pregnant woman: a case observed with a steady-state free precession imaging sequence. Magn Reson Imaging 2002, 20:301-304.

21. Iwamoto H, Suzuki M, Watanabe N, Minai M, Hirata S, Hoshi K: Case study of a pregnant woman with decidualized ovarian endometriosis whose preoperative findings suggested malignant transformation. Eur J Gynaecol Oncol 2006, 27:301-303.

22. Machida S, Matsubara S, Ohwada M, Ogoyama M, Kuwata T, Watanabe T, Izumi A, Suzuki M: Decidualization of ovarian endometriosis during pregnancy mimicking malignancy: report of three cases with a literature review. Gynecol Obstet Invest 2008, 66:241-247.

23. Takeuchi M, Matsuzaki K, Nishitani H: Magnetic resonance manifestations of decidualized endometriomas during pregnancy. J Comput Assist Tomogr 2008, 32:353-355.

24. Yoshida S, Onogi A, Shigetomi H, Tsuji Y, Haruta S, Naruse K, Kanayama S, Noguchi T, Sakata M, Furukawa N, Sado T, Yamada Y, Kitanaka T, Oi H, Kobayashi $\mathrm{H}$ : Two cases of pregnant women with ovarian endometrioma mimicking a malignant ovarian tumor. J Clin Ultrasound 2008, 36:512-516.

25. Tazegül A, Seçilmiş Kerimoğlu O, Incesu FN, Doğan NU, Yılmaz SA, Celik C: A Case Presentation: Decidualized Endometrioma Mimicking Ovarian Cancer during Pregnancy. Case Rep Obstet Gynecol 2013. doi:10.1155/2013/728291.

26. Fruscella E, Testa AC, Ferrandina G, Manfredi R, Zannoni GF, Ludovisi M, Malaggese M, Scambia G: Sonographic features of decidualized ovarian endometriosis suspicious for malignancy. Ultrasound Obstet Gynecol 2004, 24:578-580

27. Meresman GF, Auge L, Baranao Rl, Lombardi E, Tesone M, Sueldo C: Oral contraceptives suppress cell proliferation and enhance apoptosis of eutopic endometrial tissue from patients with endometriosis. Fertil Steril 2002, 77:1141-1147.

28. Davis L, Kennedy SS, Moore J, Prentice A: Modern combined oral contraceptives for pain associated with endometriosis. Cochrane Database Syst Rev 2007, 18:CD001019. Review.

29. Speroff L, Fritz MA: Clinical Gynecologic Endocrinology and Infertility 8th ed. Philadelphia, USA: Lippincott Williams \& Wilkins; 2010.

30. Sammour RN, Leibovitz Z, Shapiro I, Degani S, Levitan Z, Aharoni A, Tal J, Lurie M, Ohel G: Decidualization of ovarian endometriosis during pregnancy mimicking malignancy. J Ultrasound Med 2005, 24:1289-94.

31. Whitecar MP, Turner S, Higby MK: Adnexal masses in pregnancy: a review of 130 cases undergoing surgical management. Am J Obstet Gynecol 1999, 181:19-24.

32. Wilasrusmee C, Sukrat B, McEvoy M, Attia J, Thakkinstian A: Systematic review and meta-analysis of safety of laparoscopic versus open appendicectomy for suspected appendicitis in pregnancy. Br J Surg 2012, 99:1470-8.

33. Harrison P: Psychosocial impact of a cancer diagnosis during pregnancy. Nurs Womens Health 2013, 17:437-42.

34. Holland TK, Cutner A, Saridogan E, Mavrelos D, Pateman K, Jurkovic D: Ultrasound mapping of pelvic endometriosis: does the location and number of lesions affect the diagnostic accuracy? a multicentre diagnostic accuracy study. BMC Women's Health 2013, 13:43. doi:10.1186/1472-6874-13-43.

35. Aslam N, Ong C, Woelfer B, Nicolaides K, Jurkovic D: Serum CA125 at 11-14 weeks of gestation in women with morphologically normal ovaries. BJOG 2000, 107:689-690.

doi:10.1186/1472-6874-14-128

Cite this article as: Pateman et al:: Natural history of ovarian endometrioma in pregnancy. BMC Women's Health 2014 14:128. 